\title{
Reviewers in this Issue
}

Prof. Mahmuda Begum

Department of Pharmacology \& Therapeutics

Enam Medical College, Savar, Dhaka

Prof. Iftikhar Ahmed

Department of Microbiology

Enam Medical College, Savar, Dhaka

Prof. Sheikh Salahuddin Ahmed

Department of Medicine

Bangladesh Institute of Health Sciences, Dhaka

Prof. Shamim Ara Khan Chowdhury

Department of Physiology

Enam Medical College, Savar, Dhaka

Prof. Md. Motahar Hossain Bhuiyan

Department of Ophthalmology

Enam Medical College \& Hospital, Savar, Dhaka

Prof. Md. Rezwanur Rahman

Department of Biochemistry

Delta Medical College, Dhaka

Prof. Kabir Ahmed

Department of Ear, Nose \& Throat

Enam Medical College \& Hospital, Savar, Dhaka

Dr. Irin Perveen

Associate Professor

Department of Gastroenterology

Enam Medical College \& Hospital, Savar, Dhaka

Dr. Sharmin Akhtar Rupa

Associate Professor

Department of Radiology \& Imaging

Popular Medical College \& Hospital, Dhaka 